\title{
Archéopages
}

Archéopages

Archéologie et société

Hors-série 3 | 2012

Nouveaux champs de la recherche archéologique

\section{La part d'héritage : le cas des céramiques pérennes d'époque moderne et contemporaine}

Alban Horry

\section{(2) OpenEdition}

1 Journals

Édition électronique

URL : https://journals.openedition.org/archeopages/727

DOI : 10.4000/archeopages. 727

ISSN : 2269-9872

Éditeur

INRAP - Institut national de recherches archéologiques préventives

Édition imprimée

Date de publication : 1 janvier 2012

Pagination : 188-193

ISSN : $1622-8545$

\section{Référence électronique}

Alban Horry, "La part d'héritage : le cas des céramiques pérennes d'époque moderne et

contemporaine », Archéopages [En ligne], Hors-série 3 | 2012, mis en ligne le 01 janvier 2012, consulté

le 25 février 2023. URL : http://journals.openedition.org/archeopages/727 ; DOI : https://doi.org/

$10.4000 /$ archeopages. 727 


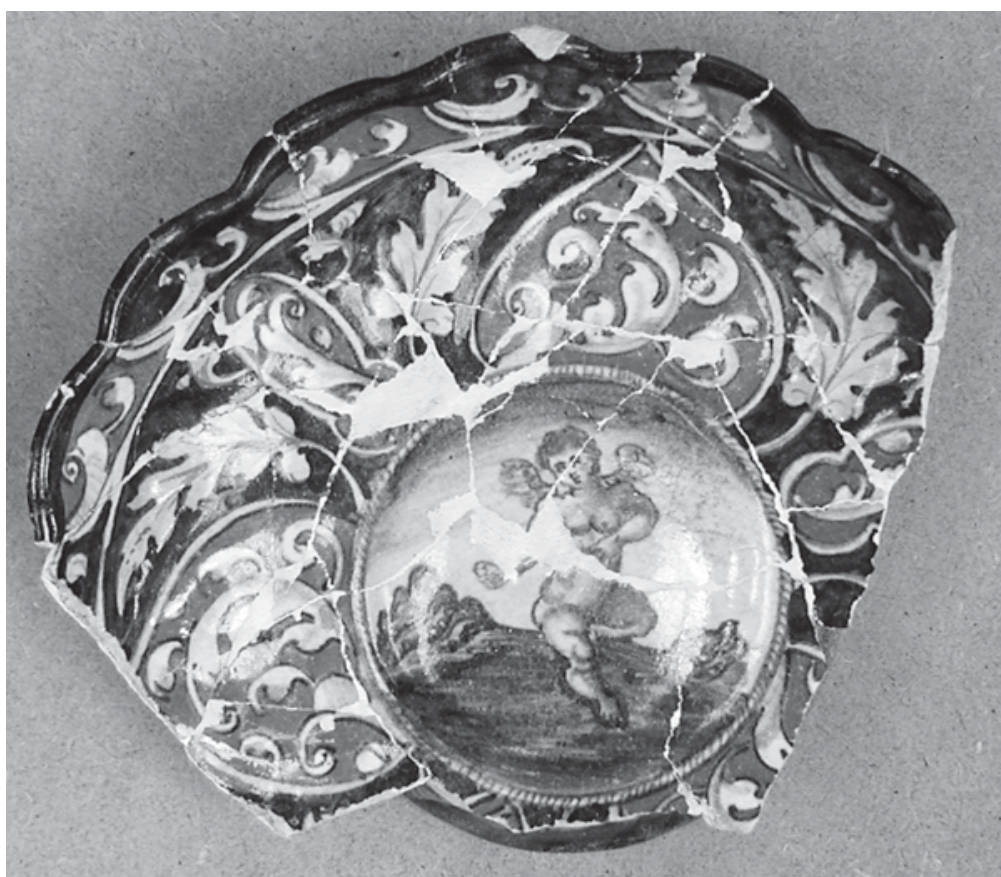

[Fig.1] Coupe à godrons ou crespina en majolique de Faenza, milieu du $\mathrm{XVI}^{\mathrm{e}}$ siècle. Lyon. Fouilles de la place de la Bourse.

[Fig.2] Assiette en faïence de Varages (Var) avec agrafe de restauration. Lyon, Fouilles du Parc Saint-Georges.

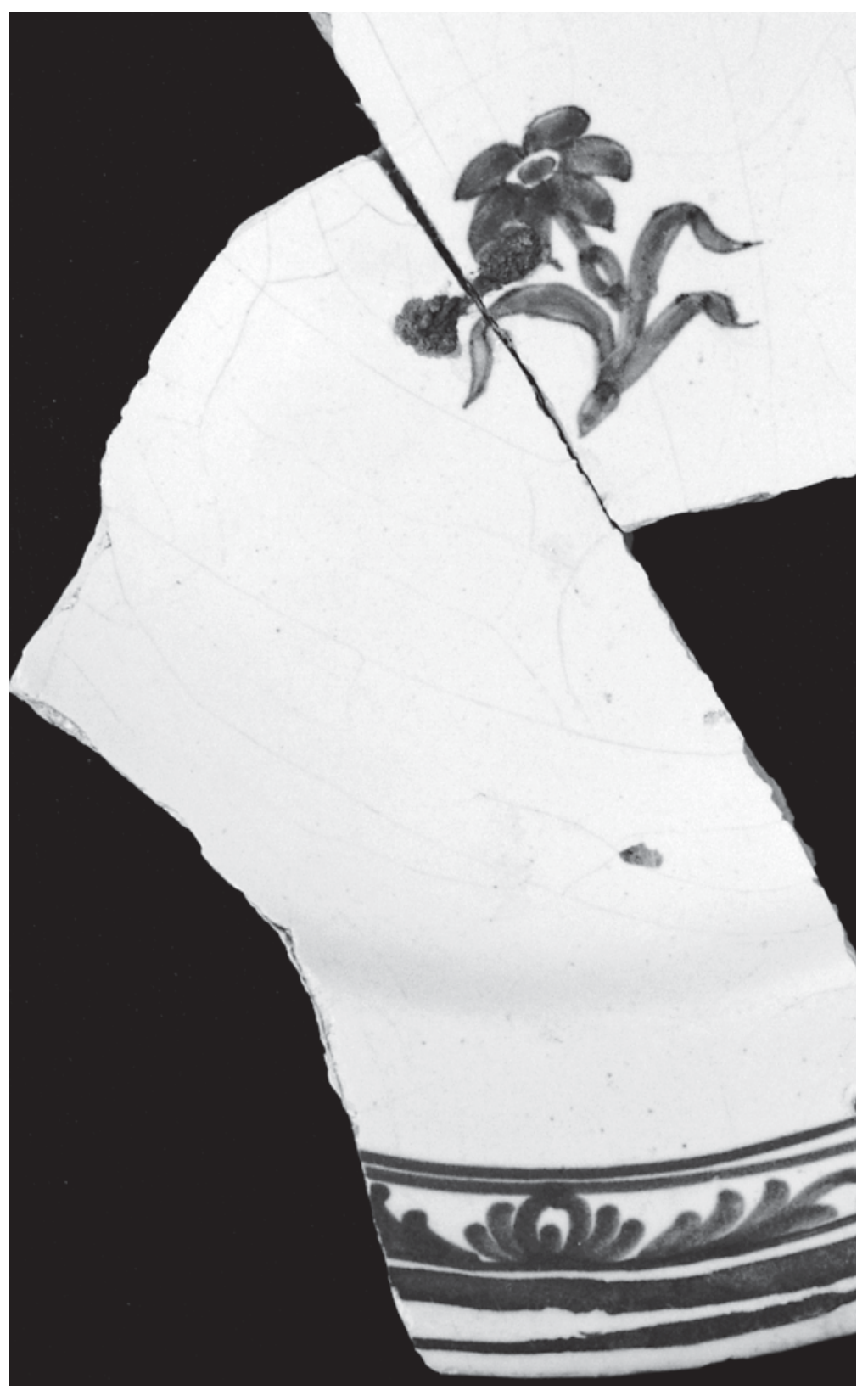




\section{La part d'héritage : le cas des céramiques pérennes d'époque moderne et contemporaine}

Alban Horry

Inrap, UMR 5138 "Archéométrie et archéologie »

$\mathbf{L}$ essor de l'archéologie des temps modernes en région Rhône-Alpes a été rapidement associé au développement des études céramologiques à partir de la fin des années 1980. Auparavant, les céramiques de lépoque moderne étaient surtout étudiées par des historiens des arts décoratifs ou ethnologues et ce, dès la fin du XIX ${ }^{\mathrm{e}}$ siècle. Il faudra attendre le début des années 1980 pour que les divers aspects des vaisseliers régionaux soient abordés par l'archéologie. L'application des méthodes de l'archéologie préventive aux sites des $\mathrm{XVIII}^{\mathrm{e}}$ et $\mathrm{XIX}^{\mathrm{e}}$ siècles est aussi remarquable dans la région Rhône-Alpes, mais elle est finalement assez récente. C'est d'ailleurs ce sujet qui avait fait l'objet d'un article par Élise Faure-Boucharlat dans la revue Les nouvelles de l'archéologie en 2005 (Faure-Boucharlat, 2004). Le souvenir de périodes peu fastes, où les mobiliers archéologiques postérieurs à l'Antiquité ou au Moyen Âge, étaient jetés sans étude, reste encore gravé dans la mémoire des archéologues. Il semble qu'aujourd'hui la situation soit un peu différente, mais il reste encore parfois nécessaire de convaincre de lintérêt des périodes dites « récentes ».

L'héritage de 30 ans de céramologie moderne. L'étude des poteries de la période moderne est motivé par la création, à Lyon, en 1985, d'un groupe de travail exclusivement consacré à l'étude des céramiques médiévales et post-médiévales, qui fixe les bases des axes de recherche sur les vaisselles en terre cuite produites et consommées en Lyonnais et Dauphiné. Ce projet collectif de recherche, «les ateliers médiévaux et modernes, l'archéologie industrielle, organisation et diffusion » regroupait des archéologues et des céramologues en collaboration avec le Laboratoire de céramologie de Lyon. En effet, le recours à l'archéométrie devint alors essentiel afin de définir les aires de production et de diffusion des vaisselles des XVII ${ }^{\mathrm{e}}$ et XVIII ${ }^{\mathrm{e}}$ siècles. Ces travaux aboutiront à la réalisation de deux publications importantes : le catalogue de

1 Ce dernier volume associait des études de cas, comme la

localisation de certains

ateliers de la fin

du Moyen Âge ou

de la période moderne,

mais également une

partie proposant une

première approche

typologique du répertoire

des vaisselles domestiques lyonnaises.

2 La mise en place

du répertoire des formes

pour la période

$\mathrm{XVI}^{\mathrm{e}}$-XIX ${ }^{\mathrm{e}}$ siècle en région

Rhône-Alpes est en cour

d'élaboration dans

le cadre d'un futur numéro de la collection

Documents d'Ar
en Rhône-Alpes.

3 Responsable : C. Arlaud

(Afan). l'exposition À la fortune du pot en 1990 (Collectif, 1990) et le volume 12 des Documents d'Archéologie en Rhône-Alpes en 1996 $6^{\mathbf{1}}$ (Faure-Boucharlat et al., 1996). La masse de mobilier accumulée depuis, grâce aux nombreuses opérations d'archéologie préventive, est à l'origine de la constitution d'ensembles de référence. Un dépouillement des données, effectué dans le cadre d'un diplôme universitaire en 2005-2006, permet d'évoquer près de 500.000 tessons de céramique pour des lots allant du XVI ${ }^{\mathrm{e}}$ au XIX ${ }^{\mathrm{e}}$ siècle, provenant d'un vaste territoire compris entre Savoie, Dauphiné, Lyonnais et Forez. Tous les types de contextes «sociaux» et artisanaux sont renseignés et donnent aujourd'hui une image assez précise des objets du quotidien en terre cuite entre la fin du Moyen Âge et le début de la période contemporaine (Horry, 2010 et 2011).

Il apparaît évident, au vu des collections disponibles aujourd'hui, que les périodes les plus récentes, c'est-à-dire les XVIII ${ }^{\mathrm{e}}$ et XIX ${ }^{\mathrm{e}}$ siècles, vont fournir progressivement des éléments inédits dans l'analyse archéologique du vaisselier. La qualité et la quantité d'informations livrées par cette masse mobilière est remarquable. La constitution de lots de plusieurs dizaines de milliers de fragments de vases divers offre l'opportunité de travailler sur un échantillonnage cohérent et pertinent, reflétant sans doute presque tout l'éventail des vaisselles consommées et donc utilisées au quotidien.

Les méthodes de comptage des études céramologiques de lots modernes ne diffèrent pas, ou très peu, de ce qu'il est commun d'observer pour les autres périodes chronologiques avec la détermination d'un nombre de tessons et d'un nombre minimum d'individus utiles pour établir des statistiques. Ces dernières sont utilisées pour cerner l'apparition ou la disparition de tel ou tel groupe technique, afin de donner en premier lieu une information utile à la chronologie dans la mise en forme des données de terrain. Le fait que la céramologie des périodes moderne et contemporaine soit récente est un élément important qui doit être pris en compte (Journot, Bellan, 2011, p. 93-116). En effet, l'origine de plus de la moitié des productions diffusées entre les $\mathrm{XVI}^{\mathrm{e}}$ et $\mathrm{XIX}^{\mathrm{e}}$ siècles est inconnue et ces dernières sont, pour leur majorité, aujourd'hui classées en fonction d'observations macroscopiques. D'autres problèmes se posent pour la mise en place de typologies périodisées. En effet, la diversification du répertoire, par rapport à la période médiévale, l'arrivée de nouveaux produits, soit importés, soit de production locale, parfois quasi industrielle influe de façon notable sur la constitution des vaisseliers. Le recours à des typologies assez simplifiées est nécessaire pour mettre en place un répertoire morphologique qui puisse être régulièrement enrichi de nouvelles formes et surtout facile d'utilisation pour l'archéologue confronté à des masses de mobilier². Aujourd'hui grâce à la présence de ces données abondantes et à un travail continu depuis un peu plus de vingt ans, les bases du vaisselier domestique lyonnais en particulier sont fixées ; il reste encore de très nombreux points d'ombre, surtout dans 


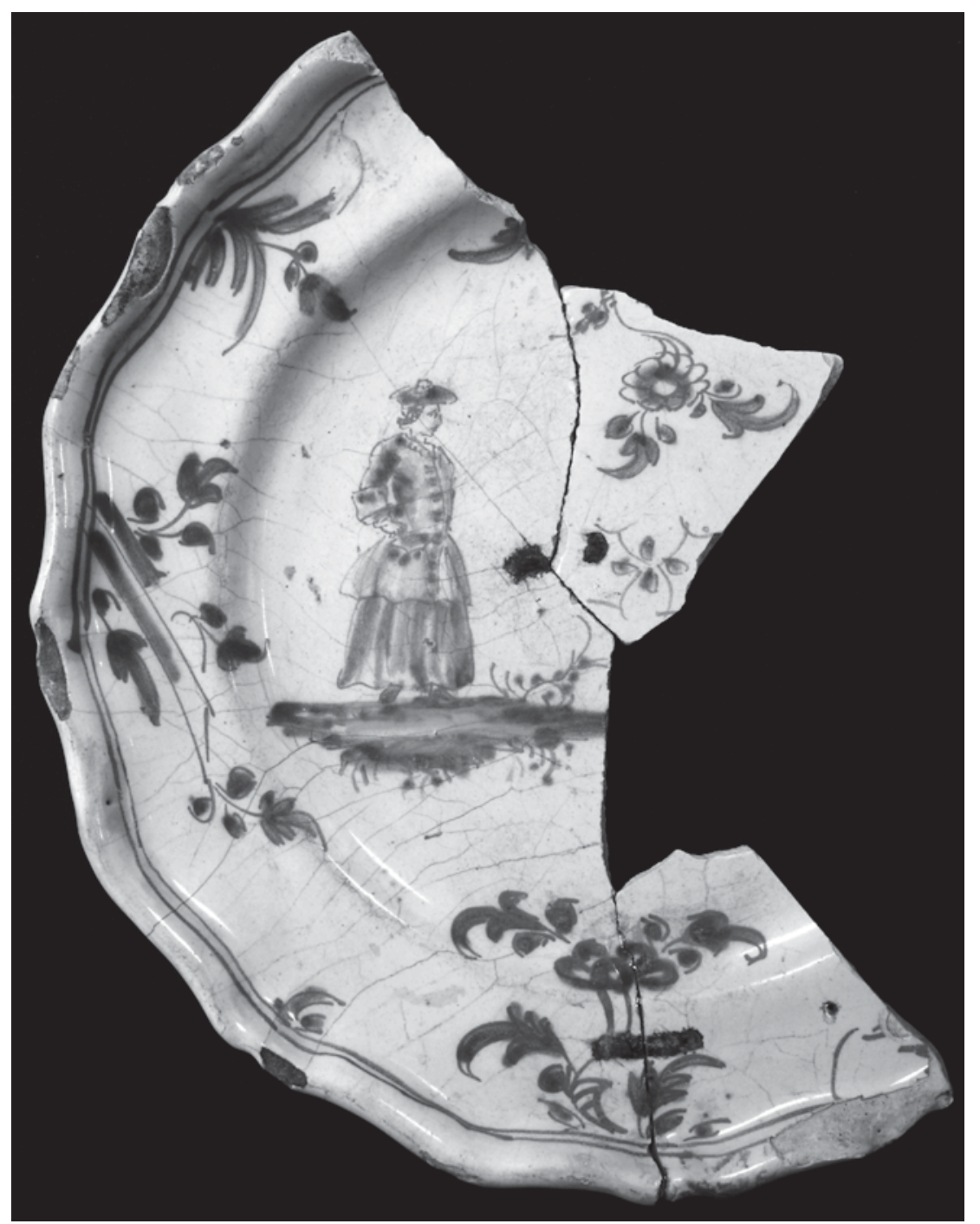

[Fig.3] Assiette au décor vert et orange en faïence de Lyon avec agrafes de restauration. Lyon. fouilles du Parc Saint-Georges.

[Fig.4] Mortier en céramique engobée, glaçuré, avec agrafes de restauration. Lyon, fouilles du Parc Saint-Georges.

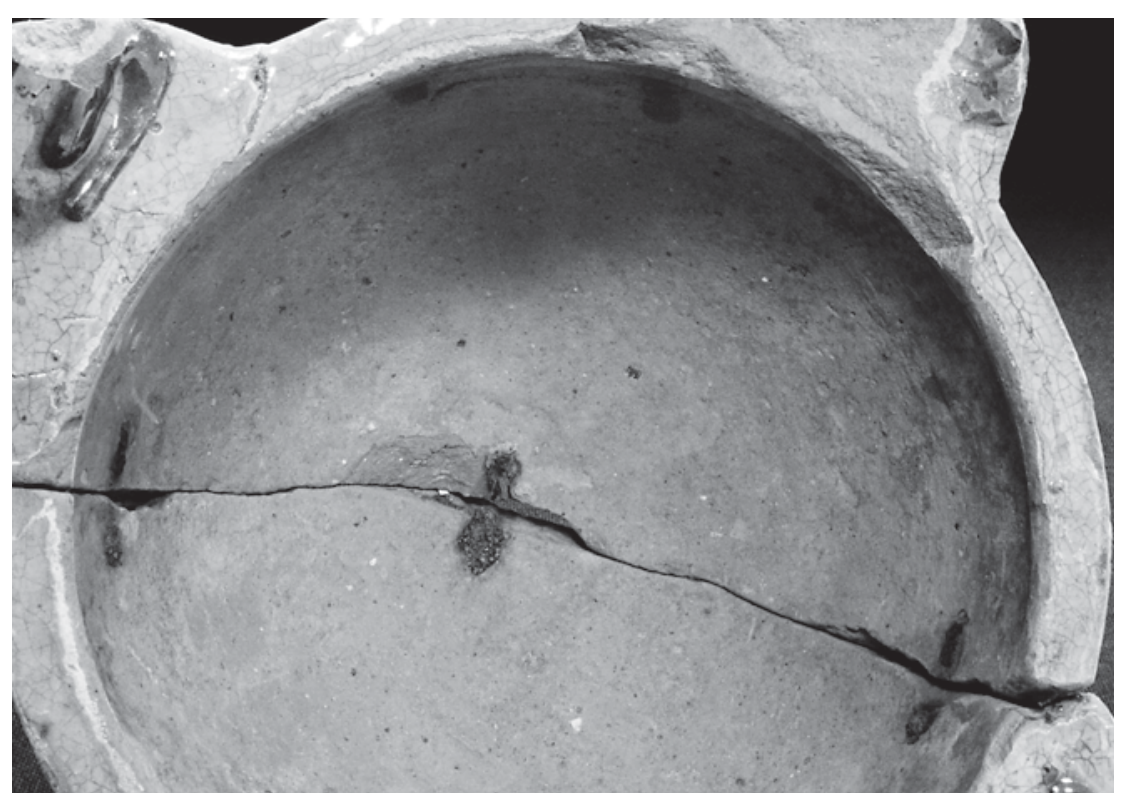


l'identification des origines de produits.

Les contextes de dépotoirs domestiques sont assurément les plus «parlants» et offrent l'opportunité de s'extraire du cadre strict de la datation archéologique et de la typologie. L'étude en cours d'un lot singulier de la fin du XVIII siècle, découvert sur le site du Parc Saint-Georges à Lyon, offre l'opportunité de réfléchir sur certains aspects particuliers. Sans faire cependant des généralités des phénomènes observés, il est intéressant de s'arrêter un instant sur une identité particulière qui affecte la constitution de certains lots régionaux de céramiques entre les $\mathrm{XVI}^{\mathrm{e}}$ et $\mathrm{XIX}^{\mathrm{e}}$ siècles : la présence, au sein d'ensembles datés précisément, d'éléments bien plus anciens de quelques dizaines d'années et qui témoignent de la valeur d'usage ou affective données à quelques céramiques.

\section{Le dépotoir d'une riche demeure lyonnaise} au début du XVII ${ }^{\mathrm{e}}$ siècle. Le premier ensemble dans lequel ce phénomène peut être observé est un dépotoir sur la place de la Bourse à Lyon, fouillée en $1991^{3}$ (Arlaud et al., 2000). Le secteur de la découverte correspond aux jardins situés à l'arrière des maisons et a livré une zone de rejet sous la forme d'une grande fosse sans aménagements particuliers. Un mobilier céramique de qualité, que l'on peut assez précisément dater de l'extrême fin du XVI ${ }^{\mathrm{e}}$ siècle ou du tout début du XVII ${ }^{\mathrm{e}}$ siècle, permet d'effectuer de nombreuses observations sur le vaisselier. Caractérisé par une réelle diversification du répertoire, il présente toutes les innovations connues en ce début de période moderne. Les céramiques à pâte engobée et glaçurée connaissent alors un essor particulier avec la vaisselle de table individuelle et les très nombreuses écuelles à oreilles, aux décors variés. La vaisselle culinaire n'est désormais presque représentée que par les productions des ateliers de Meillonnas et Treffort. Les céramiques communes grises régressent considérablement par rapport au XVI ${ }^{\mathrm{e}}$ siècle et ne concernent plus alors que quelques vases comme les chauffe-plats ou les lèchefrites. Mais, ce qui nous intéresse tout particulièrement dans cet ensemble, c'est la découverte de vaisselles que l'on pourra qualifier ici de luxueuses, les faïences (Horry, 2001, p. 150-154). Si leur présence est tout à fait logique à cette période, les vases découverts constituent deux ensembles qui méritent attention. En premier lieu, on citera des productions qui appartiennent assurément à la fin du XVI ${ }^{\mathrm{e}}$ ou au début du XVII ${ }^{\mathrm{e}}$ siècle, comme les trois récipients, drageoirs et cuvette, en faïence blanche aux décors a compendiario, attribués à Lyon par des analyses de pâtes. Il en est de même pour un albarello dit «à double bulbe» d'origine lyonnaise et dont la diffusion est aussi placée au début du XVII ${ }^{\mathrm{e}}$ siècle et pour un grand vase d'autel à têtes de griffons, uniformément blanc, peut être d'origine nivernaise. Enfin, une coupe à godrons, ou crespina en italien, ornée d'un motif floral et animalier bleu sur blanc, évoque les productions de Montelupo du tout début du XVII ${ }^{\mathrm{e}}$ siècle. Le second ensemble présente, pour sa part, des productions lyonnaises, mais cette fois-ci antérieures au milieu du XVI ${ }^{\mathrm{e}}$ siècle et une crespina
[Fig.1] sans doute originaire de Faenza en Italie et richement décorée à l'aide du motif a quartieri, production attestée à partir des années 1530-1540. Ce lot présente, associées, des productions distantes d'une cinquantaine d'années et cette observation peut seulement être effectuée sur la base des vaisselles émaillées. Le luxe de certaines est assurément à l'origine de leur conservation sur une durée assez longue. Ces pièces brouillent les pistes quant à leur aptitude à fournir une datation précise ; en effet il faut utiliser avec prudence leur présence dans certaines structures. L'exemple de la crespina de Faenza est significatif car cette dernière, produite aux alentours de 1540, se retrouve alors dans un dépôt daté d'une cinquantaine ou d'une soixantaine d'années plus tard. Elle fournit alors un terminus post quem à ce dépotoir au même titre que les productions lyonnaises de la première moitiédu $\mathrm{XVI}^{\mathrm{e}}$ siècle. La datation du lot est confirmée par la présence de numéraire de l'extrême fin du XVI ${ }^{\mathrm{e}}$ siècle et par certaines productions en céramique engobée qui sont connues seulement à partir du début du $\mathrm{XVII}^{\mathrm{e}}$ siècle. Certes, on ne peut pas encore parler ici de conservation de vases sur une très longue durée, mais de pièces luxueuses qui ont eu, sans doute en raison de leur utilisation décorative et non pas culinaire, une vie un peu plus longue.

Traces d'une pharmacie hospitalière. Pour parler de longue durée on peut se référer à une découverte effectuée à Feurs (Loire) en 2010. Une fouille préventive 4 , menée sur l'emplacement d'un ancien hôpital a permis de découvrir un petit lot de céramiques provenant d'une fosse-dépotoir. Les différents groupes techniques découverts sont caractéristiques des vaisselles utilisées en Lyonnais et dans le Forez au tout début du XviII ${ }^{\mathrm{e}}$ siècle [Fig. 2]. On trouve ainsi des productions originaires des ateliers bressans de Meillonnas-Treffort (Ain) et des céramiques à pâte calcaire engobée et glaçurée qui représentent l'essentiel des vaisselles. On mentionnera quelques faïences avec un pot canon et un albarello. Si le premier évoque les productions de Nevers diffusées dans le courant du XVII ${ }^{e}$ siècle, l'albarello est intéressant à plus d'un titre. Il s'agit en effet d'une production originaire de Lyon et fabriquée dans des ateliers tenus par des potiers italiens vers le milieu du $\mathrm{XvI}^{\mathrm{e}}$ siècle. Ce récipient est orné d'un décor végétal polychrome associant un motif de feuilles et de grosses rosaces; sa présence dans ce secteur du Forez constitue en outre un point supplémentaire sur la carte de diffusion de ces produits. Il est davantage troublant qu'il soit découvert mêlé à des rejets du début du XVIII ${ }^{\mathrm{e}}$ siècle. Il illustre assez clairement la conservation d'objets très particuliers sur plusieurs siècles. On l'a vu, l'emplacement de la découverte est un petit dépotoir sans doute lié à l'activité hospitalière, et les vaisselles domestiques sont liées à la consommation des mets par les malades, les écuelles individuelles et les petits récipients pour réchauffer, comme les coquemars, en témoignent. La présence de plusieurs pots destinés à la conservation de denrées ou de produits d'apothicairerie est un second élément marquant et qui est bien sûr lié au contexte hospitalier.

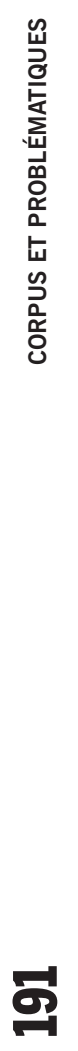

$\Phi$ 
La présence de l'albarello en faïence de Lyon, fabriqué au milieu du XVI ${ }^{\mathrm{e}}$ siècle, dans un dépotoir de la première moitié du XVIII ${ }^{\mathrm{e}}$ siècle, illustre la conservation de tels objets, en raison de leur utilisation spécialisée dans les pharmacies hospitalières. On peut mentionner pléthore de collections encore visibles de nos jours comme celle de l'Hôtel-Dieu de Lyon ou encore les apothicaireries de Louhans ou Troyes, qui possèdent, dans leurs collections, de semblables vases. Plus récemment, on peut mentionner également la découverte de pots de pharmacie lyonnais du XVI ${ }^{\mathrm{e}}$ siècle dans un dépotoir de couvent à Épinals. Dans le cas de Feurs, on a donc plutôt affaire à un récipient particulier conservé sans doute au sein d'une pharmacie hospitalière.

Dans les maisons des canuts de Saint-Georges. Enfin, un autre ensemble s'avère très pertinent pour illustrer ce phénomène de céramiques pérennes : celui issu des fouilles du parc SaintGeorges $^{6}$ (Ayala, à paraître). On s'arrêtera en particulier sur le lot daté de la fin du XviII ${ }^{\mathrm{e}}$ siècle, constitué de près de 60 ooo tessons, provenant de dépotoirs situés sur les berges de la Saône, dans un secteur portuaire et au pied des maisons construites directement au bord de la rivière. L'étude d'un lot aussi complet est révélateur des changements qui affectent le vaisselier moderne à cette période (Horry, 2012). Outre la possibilité de constituer un répertoire quasi complet et de fixer la typo-chronologie des vaisselles lyonnaises à la fin de la période moderne, une telle masse permet aussi d'observer certains aspects relatifs à l'utilisation des objets domestiques en terre cuite. Il en est ainsi des nombreuses traces d'utilisations ou stigmates révélant parfois des usages détournés. L’exemple le plus parlant est la cruche ou l'assiette couverte de traces de suie révélant une disposition sur ou près du feu pour des vaisselles au départ prévues pour la table. L'inventaire de ce lot a révélé également de nombreuses restaurations sur divers récipients. Cette rénovation se trouve matérialisée majoritairement par la présence d'agrafes métalliques ou par leurs traces sur de nombreux vases. Il est clair que les faïences sont de loin les plus affectées. Souvent, on peut observer des assiettes brisées en plusieurs morceaux qui connaissent une seconde vie grâce à ces petits fragments de fer. Cela correspond au départ à une volonté de conservation de pièces décorées, esthétiques, qui participent à l'embellissement de la maison, ou bien issues de quelque héritage ou cadeau et qui portent alors une certaine valeur affective. Dans ces maisons du bord de Saône, comme sans doute partout ailleurs, on tient à ses pots. Ces restaurations sur de belles assiettes de Moustiers, Varages ou Lyon [Fig. 3] se retrouvent aussi parfois sur d'autres, sans doute de moindre prix, comme celles, plus sobres, en faïence blanche sans décor et sans doute de moindre coût. Il en est ainsi également pour des récipients à vocation culinaire et on voit de grosses agrafes disposées sur des pots à cuire ou des daubières en céramique d'Ettrepigney par exemple (productions diffusées à Lyon au XVIII ${ }^{e}$ et XIX $X^{e}$ siècle et originaires d'ateliers situés dans le Jura). Pourquoi ne pas envisager que ces « vieux pots » étaient préférés à d'autres pour le goût particulier qu'ils pouvaient donner aux préparations culinaires (Alexandre-Bidon, 2005, p. 122-160) ? Les exemples ne manquent pas, dans notre quotidien, de récipients de cuisine que l'on préserve pour diverses raisons. On trouve aussi, dans ce dépotoir de Saint-Georges, d'autres récipients communs du quotidien, comme les mortiers qui ont été restaurés [Fig.4], mais qui, à l'issue de cette action, devaient être utilisés à d'autre fins que leur vocation première. Enfin, ces méthodes de préservation concernent également certains exemplaires bien déterminés et illustrés par la découverte, au sein de cet ensemble daté de la fin du XVIII ${ }^{\mathrm{e}}$ siècle, de céramiques bien plus anciennes. Une première vision globale du lot de faïences issues du dépotoir dessine une image très particulière, où des styles d'époques et d'origines différentes se retrouvent mêlés dans un même ensemble. C'est le cas d'une série de récipients en faïence originaires de Nevers. Ces produits, que l'on date de la fin du XVII et du début du XVIII ${ }^{\text {e }}$ siècle sont assez bien représentés sous la forme de coupes, assiettes à large marli, cruches, plats à barbes... à l'émail bleu et aux décors chinois. Sur certains, on note des agrafes de restauration, preuve indubitable d'une volonté de préservation d'objets décoratifs. On citera également cette assiette de la fin du XVII ${ }^{\mathrm{e}}$ siècle provenant peutêtre de la manufacture de Saint-Jean-du-Désert, à Marseille, et des faïences très fines provenant de Savone, en Italie, près de Gênes, elles aussi datées de la fin du XVII e siècle. On aurait pu envisager qu'on ait affaire à du mobilier issu de niveaux plus anciens, que l'on appelle « résiduel » en céramologie, et mélangé au mobilier des années 1770-178o, mais on trouve également, dans le lot, des productions issues des diverses manufactures ayant officié dès les 50 premières années du XVIII ${ }^{\mathrm{e}}$ siècle : Lyon, Nevers, Moustiers, Meillonnas, Delft... Autre élément qui peut être pris en considération : le statut social ${ }^{7}$ des habitants du quartier où est situé le dépôt céramique. Pendant la seconde moitié du XVIII ${ }^{\mathrm{e}}$ siècle, le secteur compte une population assez peu aisée, essentiellement tournée vers les travaux liés à la rivière et l'artisanat, dont, en particulier, les premiers canuts ou ouvriers de la soie avant leur départ pour le quartier de la Croix-Rousse. Ceci explique peut-être que l'on retrouve finalement peu de céramiques dites de luxe et que les rares pièces les plus prestigieuses soient régulièrement restaurées.

Une analyse détaillée de ces dépotoirs d'époque moderne invite donc à une utilisation prudente des produits prestigieux ou importés pour établir la datation qui ne peut résulter que de la conjugaison des différents groupes techniques et non pas reposer uniquement sur les pièces les plus facilement identifiables. Certes, elles fournissent un terminus chronologique pour la mise en place du dépôt, mais elles renseignent surtout sur les habitudes domestiques, au même titre que certains
7 On citera à ce titre les études réalisées par Fabienne Ravoire (Inrap) sur les rapports entre les statuts sociaux et les céramiques (Ravoire, 2007). 
types de production, pour les utilisations

culinaires par exemple. Dans le cadre très précis de l'archéologie postérieure au Moyen Âge, la notion de mobilier remanié ou résiduel semble donc plus difficile à percevoir et à déterminer. Travailler sur la période moderne doit être forcément associé aux études des archives écrites. Pour illustrer cette portion de mobilier domestique, les inventaires après décès pourraient être d'un certain secours. Les sources lyonnaises sont quasi muettes et les mentions de céramiques sont toujours anecdotiques. Contrairement aux vaisselles de verre ou d'étain, les poteries ont une simple valeur d'usage et qui écarte toute revente, d'où peut-être leur absence dans ces textes, ce qui n'est pas forcément le cas cependant dans toutes les régions. À Lyon, les données archéologiques sont presque les seules disponibles pour aborder cette notion de part d'héritage dont témoignent ces vaisselles familiales entre les XVI ${ }^{\mathrm{e}}$ et XIX ${ }^{\mathrm{e}}$ siècles.

Alexandre-Bidon D., 2005 : Une archéologie du goût. Céramique et consommation (Moyen Âge, Temps Modernes), Paris, Picard.

Arlaud C., Lurol J.-M., Savay-Guerraz S., Vérot-Bourrély A., Auger-Rahatsöz M., Ayala G., Bernardi P., Bertrand E., Bonnet C., Cécillon C., Forest V., Horry A., Le Nézet M., Plantevin C., Rolland M., Vaireaux F., Vicard T., 2000 : « Lyon, les dessous de la Presqu'île. Bourse-République-CélestinsTerreaux. Sites Lyon Parc Auto ", Documents d'Archéologie en Rhône-Alpes, $\mathrm{n}^{\circ}$ 20, Lyon.

AYAlA G. (DIR.), à paraître : La fouille du Parc Saint-Georges à Lyon. Formation et évolution d'un espace fluvial en bord de Saône, DAF.

COLleCtif, 1990 : À la fortune du pot. La cuisine et la table à Lyon et à Vienne ( $X^{e}$-XIX $X^{e}$ s.), Catalogue de l'exposition Lyon-Vienne-Mâcon, 1990-1991.

Faure-Boucharlat E., Vicard T., Maccari-Poisson B., SavayGuerraz S., 1996 : Pots et potiers en Rhône-Alpes. Époque Médiévale. Époque Moderne, DARA, 12, Lyon, Service régional de l'archéologie, DRAC Rhône-Alpes, $315 \mathrm{p}$

Faure-Boucharlat E., 2004: « Archéologie urbaine et époque moderne : quelques réflexions à partir d'expériences lyonnaises », Les nouvelles de l'archéologie, 96, p. 17-31.

Horry A., 2001 : « La faïence à Lyon du XIV ${ }^{\mathrm{e}}$ au XVI ${ }^{\mathrm{e}} \mathrm{s}$. : l'apport des fouilles récentes ", Archéologie du Midi Médiéval, t. 19, p. 137-179.

HORRY A., 2010 : «Terra incognita? Céramiques et archéologie des Temps Modernes : premier bilan et réflexions à partir de l'exemple de Lyon », in JeAn CHAPELOT (DIR.), Trente ans d'archéologie médiévale en France. Un bilan pour un avenir, Actes du IX ${ }^{\mathrm{e}}$ congrès international de la Société d'archéologie médiévale, Caen, Publications du Crahm.

HORRY A., 2011 : «Terres lyonnaises. Les objets de terre cuite au XVIII ${ }^{\mathrm{e}}$ s. », Archéologia, n ${ }^{\circ} 485$, p. 36-50.

Horry A., 2012 : Poteries de Lyon. 1500-185o. Morceaux choisis du quotidien à Saint-Georges, Lyon, éditions Lyonnaises d'Art et d'Histoire, Inrap.

Journot F., Bellan G. (DIR.), 2011 : Archéologie de la France moderne et contemporaine, Paris, La Découverte, 2011.

RAVOIRE F., 2007 : « Mobilier céramique des XVII ${ }^{\mathrm{e}}$ et XVIII ${ }^{\mathrm{e}}$., approche sociale: l'apport des fouilles archéologiques d'Île-de-France », in Collectif, Actes du Colloque Medieval Europe in Paris, Paris, 3-8 septembre 2007. Cf. http://medieval-europe-paris-2007.univ-paris1.fr
La mixité sociale au travers des études céramologiques de corpus médiévaux et modernes d'île-de-France

Fabienne Ravoire
Inrap

L a céramique constitue, avec les restes de faune, le mobilier le plus systématiquement retrouvé dans les contextes archéologiques d'habitats médiévaux et modernes. La confrontation des assemblages observés dans des sites de nature variée constitue un moyen pour comprendre la diffusion des productions sur un espace donné. L'Île-de-France, région pour laquelle les fouilles, en particulier préventives, de sites de ces périodes sont engagées depuis une quarantaine d'années, se trouve donc très bien documentée avec notamment de très grosses opérations comme à Saint-Denis (Seine-Saint-Denis), autour de la basilique, à Paris, d'abord sur le parvis de la cathédrale Notre-Dame, puis au Grand Louvre : trois opérations couvrant l'ancien château du Louvre jusqu'à l'emplacement du palais des Tuileries, soit une surface de près de 10 hectares. On compte encore, des années 1990 à 2000, quelques belles opérations dans la capitale (la cour du Collège de France, les thermes de Cluny, le boulevard Saint-Michel, sur la rive gauche, le boulevard Sébastopol et plusieurs parcelles bâties dans le quartier des Halles et du Marais, sur la rive droite). Depuis, les opérations se portant moins dans Paris que dans de nombreuses villes de la région, comme Meaux et Lagny (Seine-et-Marne) à l'est, Étampes (Essonne) au sud, Argenteuil à l'ouest (Val-d'Oise). Des interventions dans des milieux particulièrement prestigieux comme le château des Tuileries (appartements des proches de la reine), le château de Versailles (cour des Grands Communs), le château de Vincennes ou, encore en cours de fouille, le camp d'entrainement de Louis XIV à Saint-Germain, apportent leurs lots de mobiliers particulièrement représentatifs des modes de consommation dans ces contextes d'élite. Ceux-ci peuvent être comparés à des milieux plus simples comme une auberge à Étampes, une ferme à Varennes, des maisons villageoises à Roissy, Ivry, Tremblay, des maisons urbaines et péri-urbaines à Paris etc.

De ce fait, le nombre des céramiques médiévales et modernes dont nous disposons en Île-de-France pour étayer des études se situe parmi les plus importants de France. La recherche céramologique qui s'est développée depuis les années 1990 porte, comme à peu près partout en France, sur l'identification, la caractérisation et la datation des productions céramiques, mais également sur des aspects plus culturels de ce mobilier (comme la question de la fonction). Des classements plus ou moins efficients étant opérés sur la base de traces d'usages et aussi d'une plus ou moins grande proximité avec des céramiques identifiées dans les répertoires de l'ethnographie française (voir, 